\title{
Multiple Time Scales in Cataclysmic Variables: The Examples
}

\author{
Ivan L. Andronov \\ Department of Astronomy, Odessa State University, T.G. Shevchenko Park, \\ 270014 Odessa, Ukraine
}

\begin{abstract}
Decades of patrol observations of cataclysmic variables (CVs) show variations of the accretion rate also at a time scale of a few years which may be explained by a solar-type activity of the secondary and/or by orientation changes of the magnetic axis of the white dwarf in polars. Secondary photometric periods seen in TT Ari and possibly some other CVs need theoretical explanation. Irregular variability of some objects is characterized by power-law power spectra with a power index depending on the luminosity state of the system.
\end{abstract}

\section{Introduction}

Periodic variations in CVs are usually attributed to: the orbital motion of the binary (Krzeminski 1965), rotation of the magnetic white dwarf (Patterson 1979), to the irradiation variations of the secondary periodically changing with a beat period between the rotation of the white dwarf and its orbital motion (Patterson \& Price 1981) or to the precession of the eccentric accretion disk (Whitehurst \& King 1991). Cycle-to-cycle changes like the extreme ones observed by Andronov et al. $(1980,1992 \mathrm{~b})$ in AM Her and MR Ser, respectively, are usually attributed to a physical variability of the accretion structure.

Here we briefly overview the results on unusual irregular and quasi-periodic variations at other time scales.

\section{Year-scale variations}

Transitions from "high" to "low" luminosity states in polars (Hudec \& Meinunger 1976) and nova-like variables (e.g. Andronov et al. 1988) are due to accretion rate variations. They may be caused by a solar-type activity of the secondary (cf. Wenzel \& Fuhrmann 1983), irradiation (King and Lasota 1984) and/or changing orientation of the magnetic pole of the white dwarf in polars (Andronov 1987, 1993). Another appearance of the magnetic activity is an UV Cet-type flare of the secondary detected by Shakhovskoy et al. (1993a).

Year and decade-scale variations seen in MV Lyr (Andronov et al. 1988) and V795 Her (Wenzel et al. 1988) may argue for more complicated character of 
the activity possibly corresponding to a "century"-scale solar variation. Seasonal values of the outburst cycle length in dwarf novae may vary smoothly (Bianchini 1988) or abruptly (Andronov \& Shakun 1990), possibly indicating the existence of 2 sub-classes (Chinarova \& Andronov 1994). Individual cycle lengths show a bimodal distribution (Szkody \& Mattei 1984). Year-scale waves are also seen in low-mass X-ray binaries (Priedhorsky \& Holt 1987).

An "intermediate" luminosity state may also be distinguished in some stars. MV Lyr showed "excursions" to it $\left(\approx 15^{\mathrm{m}}\right)$ both from "high" $\left(\approx 13^{\mathrm{m}}\right)$ and "low" $\left(\approx 17^{\mathrm{m}}-18^{\mathrm{m}}\right)$ states (Andronov et al. 1988). "Outbursts" occurring every $\approx 400$ days were different from those observed in dwarf novae, and once a sequence of them with a few-day cycle and an exponentially decreasing height was observed.

An opposite behaviour shows IW And: periods of nearly constant brightness are rarely interrupted by periods of activity, when the brightness varies above and below the usual value of a day time-scale (Meinunger \& Andronov 1987).

\section{Week-scale beat periods}

They are observed in the systems with a photometric period close to an orbital one. BY Cam is a magnetic system with a white dwarf synchronizing with the orbital motion, and the current value of the "beat period" is $\approx 15$ days (Mason et al. 1994). Another example is TT Ari, where a 3.76 period was suggested (Semeniuk et al. 1987). However, in this system the photometric period is slightly shorter than the orbital one, and may be attributed neither to super-humps nor to the rotation of the white dwarf (Tremko et al. 1995), and its nature remains unclear.

\section{5-7 hour waves in TT Ari}

A secondary hour-scale wave with $P=4^{\mathrm{h}} 41^{\mathrm{m}}$ was suggested by Wenzel et al. (1986) based on the observations obtained during a 1986 campaign. However, the data obtained in 5 observatories in 1988 showed 4 other candidate periods, from which one "true" value is difficult to choose because the period is longer than an usual observational run (Tremko et al. 1992). To study properties of this wave, "super-long" runs starting in Japan, continued in Turkmenia and Russia, then in Europe and America are needed. Such a campaign was organized in 1994 and is planned to be continued in October during the coming years by using photometric and spectral ground-based and space observations. Particularly, in 1994, 96 hours of homogeneous observations were obtained by N.I. Dorokhov at the Dushak station of the Odessa observatory. Similar secondary waves seem to be observed in other systems (cf. Voykhanskaya 1989), and their theoretical and observational study is very important.

\section{Sub-hour QPOs}

Recent reviews on rapid variability are presented by Bruch $(1992,1995)$. Thus we briefly point out the most puzzling phenomena. Semeniuk et al. (1987) re- 
ported on the secular decrease of the cycle length in TT Ari from $27 \mathrm{~min}$ in 1961 to $17 \mathrm{~min}$ in 1985. Hollander and van Paradijs (1992) argued for a continuation of this trend in 1988 and interpreted these QPO's by interaction of the magnetosphere with an accretion disk with slowly changing parameters. Usually the cycle length of such variations undergoes cycle-to-cycle changes. Thus a best fit value significantly depends on the data set used. Tremko et al. (1995) argued for few preferred time scales, at which the peaks at the periodogram occur several (but not all) times.

\section{Power-law power spectra}

Aperiodic processes may be characterized by wide power spectra (cf. Box \& Jenkins 1970). If QPOs are seen as wide peaks superimposed on a "featureless continuum" (cf. Larsson 1985), some types of irregular variability may be characterized by a power-law shape $S(f) \propto f^{-\gamma}$. Terebizh (1992) reviewed cases $\gamma=0$ ("white noise"), 1 ("flicker noise") and 2 ("random walks"). Autoregressive processes studied on finite runs correspond to "noninteger" values of $\gamma$, as well as observations of the real stars AM Her, BZ Cam (Shakhovskoy et al. 1992, 1993b), HQ And (Andronov et al. 1992a), TT Ari (Tremko et al. 1995). Work on other stars is in progress showing the significance of the parameter $\gamma$ for description of the fast variability.

\section{7 "Shot-noise" models}

Inhomogeneities of the accretion stream cause rapid variations in CVs. The autocorrelation function (ACF) for "shot noise" is an exponent, which may be biased due to finite length of the run and subtraction of a sample mean (Sutherland et al. 1978). Detailed analysis of the influence of the trend removal in general form onto the ACF is presented by Andronov (1994).

Middleditch (1982) and Larsson (1985) reported on second-scale QPOs in accretion columns in polars, which Langer et al. (1982) interpreted as cyclic changes of the structure . Andronov (1987) proposed a model of "oscillating spaghetti". At the column base a "boiling-type" instability occurs. Such "bombardment" solutions lead to better explanation of the "soft X-ray excess" (Thompson et al. 1986).

\section{References}

Andronov I.L., 1987, Astron. Nachr. 308, 229

Andronov I.L., 1993, Odessa Astron. Publ. 6, 21

Andronov I.L., 1994, Astron. Nachr. 315, 353

Andronov I.L., Borodina I.G., Kolesnikov S.V., Pavlenko E.P., Shakhovskoy N.M., 1992a, Commun. Spec. Astron. Obs. 69, 112

Andronov, I.L., Fuhrmann, B., Wenzel, W., 1988, Astron. Nachr. 309, 39 
Andronov I.L., Pavlenko E.P., Seregina T.M., Shugarov, S.Yu., Shvechkova, N.A., $1992 b$, Proc. Stellar Magnetism, St.Petersburg, 160

Andronov I.L., Shakun L.I., 1990, ApSS 169, 237

Andronov I.L., Vasilieva S.V., Tsessevich V.P., 1980, Astron. Tsirk. 1142, 5

Bianchini A., 1988, IBVS 3136

Box G.E.P., Jenkins G.M., 1970, Time series Analysis. Forecasting and control, Holden Day, San Francisco

Bruch A., 1992, A\&A 266, 237

Bruch A., 1995, this volume, p. 288

Chinarova L.L., Andronov I.L., 1994, Padova-Abano Conf. on Cataclysmic Variables (in press)

Hollander A., van Paradijs J., 1992, A\&A 265, 77

Hudec R., Meinunger L., 1976, IBVS 1184

King A.R., Lasota J.P., 1984, A\&A 140, L16

Krzeminski W., 1965, ApJ 142, 1051

Langer S.H., Chanmugam G., Shaviv G., 1982, ApJ 252, 289

Larsson S., 1985, A\&A 145, L1

Mason P., Andronov I.L., Chanmugam G., Kolesnikov S. V., Pavlenko P., Shakhovskoy N.M., 1994, Padova-Abano Conf. on Cataclysmic Variables (in press)

Meinunger L., Andronov I.L., 1987, IBVS 3081

Midleditch J., 1982, ApJ 257, L71

Patterson J., 1979, ApJ 234, 978

Patterson J., Price C.M., 1981, ApJ 243, L83

Priedhorsky W., Holt S.S., 1987, SSR 45, 291

Shakhovskoy N.M., Alexeev I.Yu., Andronov I.L., Kolesnikov S.V.: 1993a, Proc. Cataclysmic Variables and Related Physics, Annals Israel Phys. Soc. 10, 237

Shakhovskoy N.M., Efimov Yu.S., Andronov I.L., Kolesnikov S.V., 1993b, IAU Symp. 155,407

Shakhovskoy N.M., Kolesnikov S.V., Andronov I.L., 1992, Proc. Stellar Magnetism, St. Petersburg, 148

Semeniuk I., Schwarzenberg-Czerny A., Duerbeck H., Hoffman M., Smak J., Stẹpień K., Tremko J., 1987, ApSS 130, 167

Sutherland P.G., Weiss M.C., Kahn S.M., 1978, ApJ 219, 1029

Szkody P., Mattei J.A., 1984, PASP 96, 998

Terebizh, V.Yu., 1992, Time Series Analysis in Astrophysics (in Russ.), Moscow

Thompson A.M., Brown J.C., Kuijpers J., 1986, A\&A 159, 202

Tremko J., Andronov I.L., Chinarova L.L., Kumsiashvili, M.I., Luthardt, R., Pajdosz G., Patkos L., Rößiger S., Zoła S., 1995, A\&A (subm.)

Tremko J., Andronov I.L., Luthardt R., Pajdosz G., Patkos L., Roessiger S., Zola S., 1992, IBVS 3763

Voykhanskaya, N.F., 1989, Preprint Spec. Ap. Obs.43

Wenzel W., Banny M.I., Andronov I.L., 1988, Mitt. Veränderl. Sterne 11, 141

Wenzel W., Bojack W., Critescu C., Dumitrescu A., Fuhrmann B., Götz W., Grelczyk H., Hacke G, Hudec R., Huth H., Kozhevnikov V.P., Kumsiashvili M.I., Mrkos A., Oláh K., Oprescu G., Patkós L., Peřestý R., Pfau W., Reimann H.-G., Richter G., Rößiger S., Shpychka I.V., Shult R., Stecklum B., Tóth I., Tremko J., Valníček B., Verdenet M., 1986, Prepr. Astron. Inst. Czechoslovak Acad Sci. 38

Wenzel W., Fuhrmann B., 1983, Mitt. Veränderl. Sterne, 9, 175

Whitehurst R., King A., 1991, MNRAS 249, 25 